\title{
Multimodal Medical Image Fusion Using Modified PCNN Based on Linking Strength Estimation by MSVD Transform
}

\author{
Hajer Ouerghi*, Olfa Mourali, Ezzeddine Zagrouba \\ 2 Rue AbouRayhaneBayrouni, Ariana 2080, Tunisia. \\ *Corresponding author. Tel.: +216 24856 335; email: werghi.hajer@gmail.com \\ Manuscript submitted May 15, 2017; accepted June 9, 2017. \\ doi: 10.177606/ijcce.6.3.201-211
}

Université de Tunis El Manar, Institut Supérieurd' Informatique, Research Team SIIVA- Laboratory. LIMTIC,

\begin{abstract}
In this paper, we propose a novel multimodal MRI and CT images fusion method based on Multi-resolution Singular Value Decomposition (MSVD) and Modified Pulse Coupled Neural Network (MPCNN).Firstly, the input pre-registered MRI and CT images are decomposed into high frequency (HF) and low frequency (LF) sub-bands by using the MSVD transform. Then, the MPCNN model is applied on each LF sub-bands. The proposed method can adaptively determine the linking strength of the MPCNN model. After that, LF coefficients are combined based on the output of MPCNN coefficients while HF coefficients are fused by using the maximum selection rule. Finally, the inverse MSVD is applied to reconstruct the fused image. Visual effect and objective evaluation criteria are used to evaluate the performance of our approach for nine pairs of MRI and CT images. The experimental results demonstrate that the proposed method has a better performance than other current methods.
\end{abstract}

Key words: Multimodal image fusion, PCNN, MSVD, link strength coefficient.

\section{Introduction}

Medical imaging fusion (MIF) is the process that combines relevant information from two or more images acquired by one or more modalities and at different times [1]. MIF is more suitable and more informative for human visual perception than the input images. Thus, it is widely used in several clinical applications like image-guided radiotherapy, image-guided surgery, non-invasive diagnosis, and treatment planning. The aim of MIF is to reduce redundancy by optimizing the relevant information and improve the quality and the reliability of multimodal images. In addition, MIF not only helps in diagnosing diseases, but also it reduces the storage cost if a single image is saved better than saving a lot of images. Over the years, various MIF techniques have been proposed. MIF methods are summarized in different families such as the methods based on Wavelets, the morphological methods, the methods based on Fuzzy Logic or on Neural Network [1]. Each fusion method uses different techniques which are divided into a spatial domain (like Principal Component Analysis (PCA), Hue Intensity Saturation (HIS) and BroveyTransform) and a transform domain (like Discrete Wavelet Transform (DWT), Curvelet Transform (CT) , Nonsubsampled Contoured Transform (NSCT)) [2], [3].

Kakarala and Ogunbona [4] proposed the Multi-resolution Singular Value Decomposition (MSVD) from the idea of singular value decomposition. MSVD transform allows decomposing of image into low pass and high pass sub-bands. Due to the multi-resolution property, MSVD is used for several multi-resolution signal and image processing like image fusion [5], [6] and image denoising [7]. In [8], image fusion method based 
on MSVD is proposed. It gives an efficient result better than to wavelets. In addition, it is easy to implement, computationally very simple, and it does not have a fixed set of basis vectors such as the wavelet and the Discrete Cosine transform (DCT).

The pulse-coupled neural network (PCNN) is a biologically inspired neural network. It has the character of global coupling and pulse synchronous of neurons. In the recent literature, several works have developed many image fusion algorithms based on PCNN model which provide good results [9], [10]. In contrast, they have two disadvantages. The first one is that the value of a single pixel is employed to motivate the neuron, whereas source images are sensitive to directional features and edges. For this reason, a pure use of single pixels is not enough. Recently, several decomposition transforms are combined with the PCNN model in image fusion applications such as DWT-MPCNN [11], Shearlet-PCNN [12] and NSCT-Multichannel PCNN [13]. The second one is that the basic PCNN model suffers from computational complexity due to a large number of parameters which makes it incompatible with MIF applications. One solution of this problem is to modify the PCNN model and estimated parameters. Xu et al. [14] presented a review of parameter optimization methods of the PCNN model, but all of them depend on the type of the application. In addition, the linking strength coefficient of the basic PCNN model has a great effect in image fusion techniques. It determines the interaction between the current neuron and surrounding neurons. Usually, it is considered as a fixed value in order to simplify the computation. However, it is an efficient way for MIF applications. So, an adaptative automatic linking strength is proposed to improve the performance of the proposed method. It is estimated by using the local low frequency coefficients value obtained by applying the MSVD transform.

This paper is organized as follows. In the first section, we present the basic PCNN process. Then, we introduce the proposed method. Thereafter, we evaluate our approach in order to shed the light on its abilities. Finally, we will summarize the major points that we have highlighted in the different parts of this paper.

\section{PCNN Model}

The PCNN model is used in several image processing applications such as the image segmentation, the image denoising, the feature extraction, the pattern recognition, and the image fusion [9]-[15]. It has two distinguishing points. The first one is that neurons which are associated with each group of spatially connected pixels with similar intensities tend to pulse together. The second one is the threshold decreases the other neurons exponentially.

The PCNN model consists of three parts: dendric tree, linking modulation and pulse generator, as shown in Fig. 1. The dendric tree is the input part that is composed of two kinds of inputs called the linking and the feeding channels. The difference between these inputs resides in the fact that the linking connections receive external stimulus.In addition, they have a faster characteristic response time constant than the feeding connections that receive external and local stimulus. The PCNN model is described by the following expressions of the $\mathrm{n}^{\text {th }}$ iteration:

$$
\begin{gathered}
F_{i, j}[n]=e^{\left(-\alpha_{F}\right)} F_{i, j}[n-1]+V_{F} \sum_{k, l} M_{i, j, k, l} Y_{i, j}[n-1]+S_{i, j} \\
L_{i, j}[n]=e^{\left(-\alpha_{L}\right)} L_{i, j}[n-1]+V_{L} \sum_{k, l} W_{i, j, k, l} Y_{i, j}[n-1] \\
U_{i, j}[n]=F_{i, j}[n]\left(1+\beta_{i, j} L_{i, j}[n]\right)
\end{gathered}
$$




$$
\begin{aligned}
T_{i, j}[n] & =e^{\left(-\alpha_{T}\right)} T_{i, j}(n-1]+V_{T} Y_{i, j}[n-1] \\
Y_{i, j}[n] & = \begin{cases}1, & U_{i, j}[n]>T_{i, j}[n] \\
0, & \text { otherwise }\end{cases}
\end{aligned}
$$

In the above expressions, $F$ and $L$ are the feeding and linking components respectively, $i$ and $j$ refer to the pixel location in the image, $k$ and $l$ refer to the dislocation in a symmetric neighborhood around one pixel, $n$ refers to current iteration, $M$ and $W$ are the constant synaptic weights, $S$ is the external stimulus, $V_{F}$ and $V_{L}$ are normalizing constants, $\alpha_{F}$ and $\alpha_{L}$ are the time constants, $V_{T}$ is the normalized constant, $\alpha_{T}$ is the time constant, $U$ is the internal state of the neuron, $\beta$ is the linking parameter, $Y$ is the output pulse of the model.

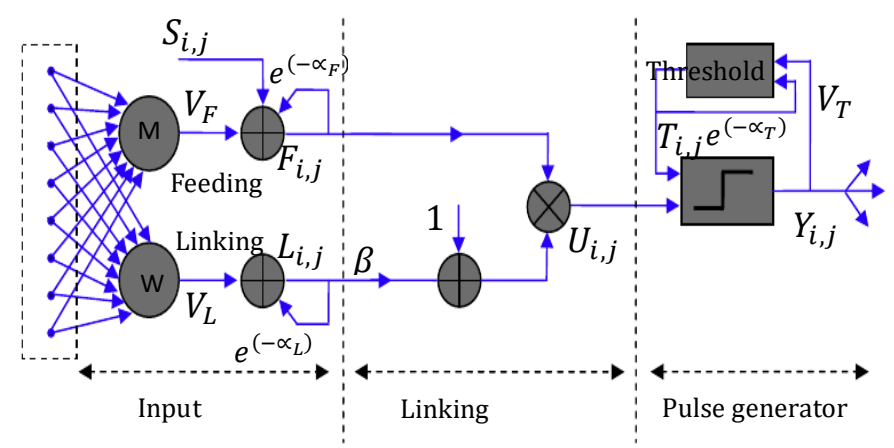

Fig. 1. PCNN model [15].

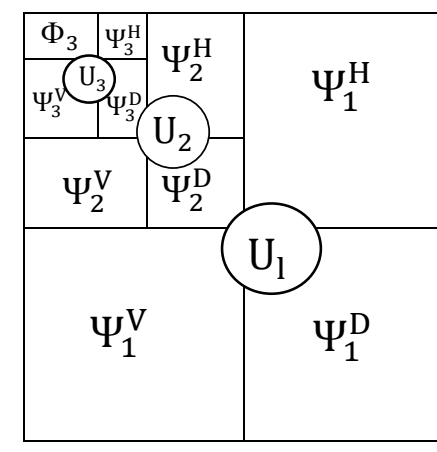

Fig. 2. MSVD structures.

\section{Proposed Method}

Multimodal computed tomography (CT) and magnetic resonance (MRI) images fusion is an important problem that is widely studied in the recent years thanks to the practical usability in clinical settings and the maturity in the technology [1]. Indeed, CT images discriminate soft tissues, blood vessels and bone structures details where MR images provide much more soft tissue information and lacks in boundary information. So, the details of CT and MRI are combined to achieve a single fused image with all relevant information.

The most important prerequisite in fusion process is the data registration. Medical images should indeed be registered in advance. In our work, the input A (CT) and B (MRI) images are already perfectly registered. The proposed method contains three parts. The first one named MSVD decomposition where the input A and B images are decomposed into high frequency (HF) and low frequency (LF) sub-bands. The second part is called fusion decision part, it groups three steps based on the modified pulse coupled neuronal networks (MPCNN). The last part named MSVD reconstruction part is based on the inverse MSVD transform. The block diagram of the proposed algorithm is shown in Fig. 3.

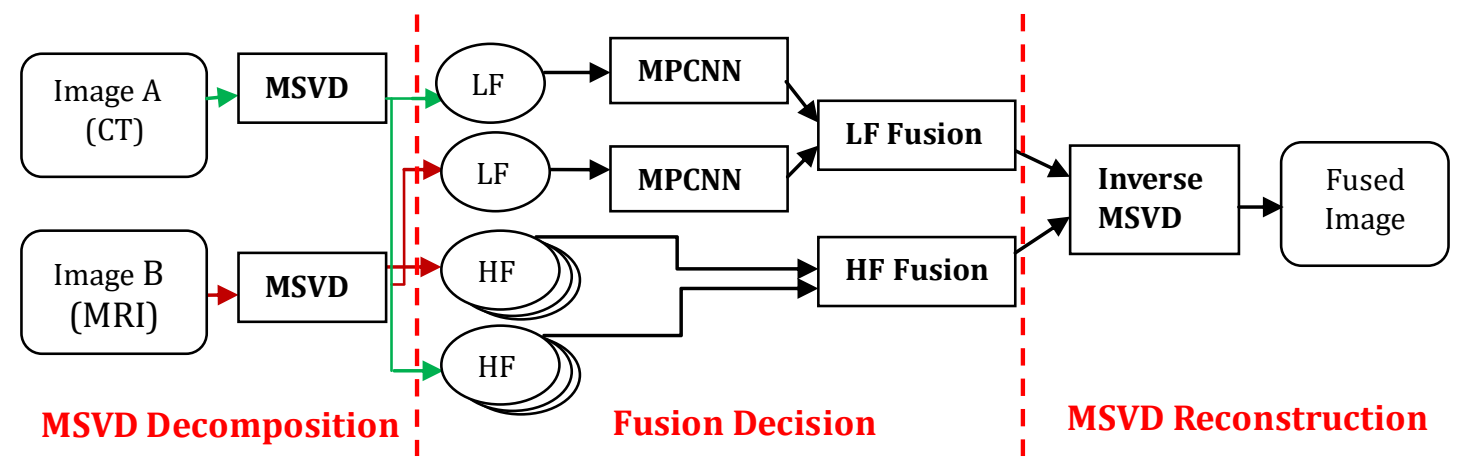

Fig.3. Block diagram of the proposed fusion method. 


\subsection{MSVD Decomposition}

Multi-Resolution Singular Value Decomposition (MSVD) is a simple process based on singular value decomposition theory [16]. It is very simple, very fast and it provides a performed fusion image [5]-[8].In our work, MSVD is used and applied for the MIF process. The concept of MSVD is similar to wavelets transform that provide directional information in decomposition levels. In MSVD, the first level of decomposition is obtained after the decimation of low and high pass filters output by a factor of two. The second level of decomposition is provided by filtering separately the decimated low pass filtered output by both filters that are followed of decimation by a factor of two. This procedure is repeated to provide successive levels of decomposition, until the desired level is obtained. In this work, the source images are decomposed in the first level of MSVD into LF and HF coefficients as represented in Fig. 3. Indeed, the input image is devised into non-overlapping $2 * 2$ blocks. Each block is arranged into a $4 * 1$ vector to form the data matrix denoted $X 1$. The blocks may be taken in transpose eigen-decomposition of the $4 \times 4$ scatter matrix $T 1$ as follows:

$$
T_{1}=X_{1} X_{1}^{T}=U_{1}^{T} S_{1}^{2} U_{1}
$$

where $X_{1}^{T}$ is the transpose vector of $X_{1}, U 1$ is the eigenvector matrix that brings $T 1$ into diagonal matrix $S_{1}^{2}$ and $U_{1}^{\mathrm{T}}$ is the transpose vector of $U_{1}$. Let $\hat{X}_{1}=U_{1}^{T} X_{1}$. The first row $\hat{X}_{1}(1,:)$ represents the LF sub-band. This approximation component corresponds to the largest eigenvalue. The other rows are the HF sub-bands that contain the detail components of an image. The elements in each row are rearranged to form $\frac{M}{2} \times \frac{N}{2}$ matrix. Let $\Phi_{1}$ denote $\frac{M}{2} \times \frac{N}{2}$ matrix formed by rearranging the row $\hat{X}_{1}(1,:)$ into matrix by first filling in the columns and then rows. Identically, each of the three rows $\hat{X}_{1}(2,:), \hat{X}_{1}(3,:)$ and $\hat{X}_{1}(4,:)$ may be arranged into $\frac{M}{2} \times \frac{N}{2}$ matrices that are denoted as $\Psi_{1}^{V}, \Psi_{1}^{H}$ and $\Psi_{1}^{D}$ respectively (Fig. 2).

\subsection{Fusion Decision}

The output decomposition coefficients of the first part are used as inputs in this part. Fusion decision part contains three steps. Firstly, the PCNN parameters are settled based on decomposition coefficients. Then, we applied the MPCNN model for only LF coefficients. Finally, the LF coefficients sub-bands are fused based on MPCNN values while HF coefficients sub-bands are fused separately based on the maximum selection rule. The basic PCNN model is a complex model due to a large number of parameters. It has also the limitation of slow processing, which makes it unsuitable for MIF applications. One solution of this problem is to modify PCNN. Our method uses the modified model according to the actual needs of image fusion [11], [17]. Fig. 4 shows the modified PCNN model. The modified expressions of the feeding and linking components are listed as follows:

$$
\begin{gathered}
F_{i, j}[n]=I_{i, j} \\
L_{i, j}[n]=\sum_{k, l} W_{i, j, k, l} Y_{i, j}[n-1]
\end{gathered}
$$

$U, T$ and $Y$ functions are computed according to (3), (4) and (5) respectively. The main advantage of the MPCNN process is that a few numbers of parameters are used. These parameters are: the link strength coefficient, the threshold amplitude coefficient and the decay time constant. There are various methods of estimation parameters [12], [17]-[20], but all of them depend on the type of the application. So, the parameters estimation constrains further the development of the PCNN model.

Step1: the initial values of MPCNN parameters are settled down. The iteration time $t$ and the $V_{L}$ parameters of the MPCNN model are set as $t=200, V_{T}=20, \alpha_{T}=0.2$ and the weight $W_{i, j}$ for each sub-band is given by: 


$$
W_{i, j}=\left(\left(i-I_{x}\right)^{2}+\left(j-I_{y}\right)^{2}\right)^{-\frac{1}{2}}
$$

where $I$ is the decomposition coefficient at the position $(x, y)$. The MPCNN model is composed of a lot of neurons. The number of neurons is equal to the number of pixels in each input image. Therefore, each Neuron represents a MSVD decomposition coefficient.

Step 2: the MPCNN model is applied for the LF sub-bands of each input image. The input neurons of the MPCNN model are computed according to (7) (8), (3), (4) and (5) respectively. In the PCNN process, the linking coefficient $\beta$ plays an important role in MIF techniques. It determines the interaction between the current neuron and surrounding neurons. Generally, in order to simplify the computation, $\beta$ is considered as a fixed value (0.2 or 0.5$)$. However, it is an efficient way for MIF applications. So, an adaptive automatic linking strength $(\beta)$ is proposed to improve the performance of our approach. For each iteration of the MPCNN, the $\beta$ coefficient is estimated. Let $\beta_{i, j}$ denote the linking strength coefficient for the neuron ij in corresponding input image. It is estimated by using the local value of LL coefficients of MSVD. It is given by:

$$
\beta_{i, j}=\sqrt{\sum_{(x, y) \in N(i, j)}\left[\left(L L_{x, y}-L L_{x+1, y}\right)^{2}+\left(L L_{x, y}-L L_{x, y+1}\right)^{2}\right]}
$$

where $N(i, j)$ is the neighborhood with the pixel $i j$ as its centre. The neighborhood size can be fixed according to the complexity extent of the image. Generally, a square region with the size $n \times n$ can be chosen where $n$ is an odd number not less than three. Furthermore, the more complex the image is, the smaller the $n$ is. So, considering the complexity, we fix in this work all the neighborhood sizes as $3 \times 3$.

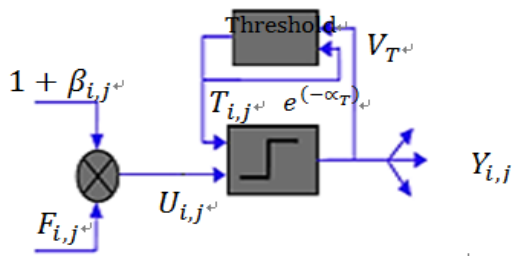

Fig.4. Modified PCNN model.

Step 3: in medical image fusion, the choice of fusion rule is very important because it affects the fusion result. Most information of the input images is kept in the low frequency sub-band which represents the approximation image. So, $L F$ coefficients are combined based on MPCNN results to find important coefficients. Let $L F_{f}(i, j)$ denoted the low frequency coefficient located at $(i, j)$ of the image. $L F_{f}$ coefficients of the fused image $F$ are obtained as follows:

$$
\begin{gathered}
\operatorname{decision}(i, j)=\left\{\begin{array}{l}
1, \operatorname{MPCNN}_{A}(i, j)>M P C N N_{B}(i, j) \\
0, \text { otherwise }
\end{array}\right. \\
F . L F_{f}(i, j)=\operatorname{decision}(i, j) \times L F_{A}(i, j)+\sim \operatorname{decision}(i, j) \times L F_{B}(i, j)
\end{gathered}
$$

On the other hand, $H F$ coefficients represent the details of the source images. The conventional way to produce the composite coefficients is to use the averaging method. However, this technique cannot only fuse the details of the high quality for medical images. So, we choose to fuse the $H F$ coefficients by using the maximum selection method. It is the largest rule used to fuse image because it can preserve more details. For each sub-band, $H F$ coefficients are fused as follow:

$$
F . H F_{f}(i, j)=\max \left(H F_{A}(i, j), H F_{B}(i, j)\right)
$$




\subsection{MSVD Reconstruction}

The final fused image is obtained from the first level of the inverse MSVD transform based on the fused low frequency $\left(F . L F_{f}\right)$ and the three fused high frequency $\left(F . H F_{f}\right)$ sub-bands.

\section{Experimental Study}

In order to evaluate the performance of the proposed method, we compare our results with other popular methods which are: Discrete Wavelet Transform (DWT) with 16 SS (2,2) [21], Gradient Pyramid (GP)[22] and NSCT combined with PCNN process(NSCT-PCNN) [17]. Several experiments were performed using nine groups of a pair of registered MRI and CT images $(256 \times 256)$ downloaded from the whole brain atlas ${ }^{1}$.

\subsection{Objective Criteria}

Besides the visual comparison between the fused image and input images, several evaluation criteria are also applied to provide an objective assessment. The selected important quantitative criteria used in the objective analysis are as follows:

Standard deviation (SD) that it is a measure of contrast in the fused images. Indeed, if the SD is higher that means a better contrast is provided.

Entropy (E) that measures the content of information in an image. It is the average number of bits needed to quantize the intensities in the image. An image with high information content would have high entropy. $E$ is defined as:

$$
E=-\sum_{g=0}^{L-1} p(g) \log _{2} p(g)
$$

where $p(g)$ is the probability of grey-level $g$, and the range of $g$ is $[0, \ldots . ., L-1]$.

Fusion Factor (FF) that provides the ability to acquire information from the input images $A$ and $B$. If the FF is higher, this means that we are obtaining a better result. The $F F$ values are defined as:

$$
\begin{gathered}
F F=M I(A, F)+M I(B, F) \\
M I(S, F)=E(S)+E(F)+E(S, F)
\end{gathered}
$$

where $M I$ is the mutual information, $E$ is the entropy, $S$ and $F$ are the source and the fused images.

Fusion Quality index $\left(Q^{A B / F}\right)$ provides the edge information preservation value. The larger the value of $Q^{A B / F}$, the better the result is. It can be calculated by:

$$
\begin{gathered}
Q^{A B / F}=\frac{\sum_{n=1}^{N} \sum_{m=1}^{M}\left(\varphi^{A F}(n, m) w^{A}(n, m)+\varphi^{B F}(n, m) w^{B}(n, m)\right)}{\sum_{n=1}^{N} \sum_{m=1}^{M}\left(w^{A}(n, m)+w^{B}(n, m)\right.} \\
\varphi^{A F}=\varphi_{g}^{A F}(n, m) \varphi_{a}^{A F}(n, m) \quad \varphi^{B F}=\varphi_{g}^{B F}(n, m) \varphi_{a}^{B F}(n, m)
\end{gathered}
$$

where $w$ is the important factor, $\varphi_{g}$ and $\varphi_{a}$ reflect the edge strength and orientation preservation values respectively.

\subsection{Results and Discussion}

Parameters of the NSCT-PCNN method were as set: $W=[0.7071,1,0.7071 ; 1,0,1 ; 0.7071,1,0.7071], \beta=$ 3, $\alpha_{T}=0.2, V_{L}=1.0, V_{T}=20$ and $T=200$.The synthesized of all images are displayed in Fig. 5. Images A and B are the source registered MRI and CT images whereas the others are the fused images obtained by the different methods (Fig. 5). Performance results are listed in Table 1, Table 2, Table 3 and Table 4. 


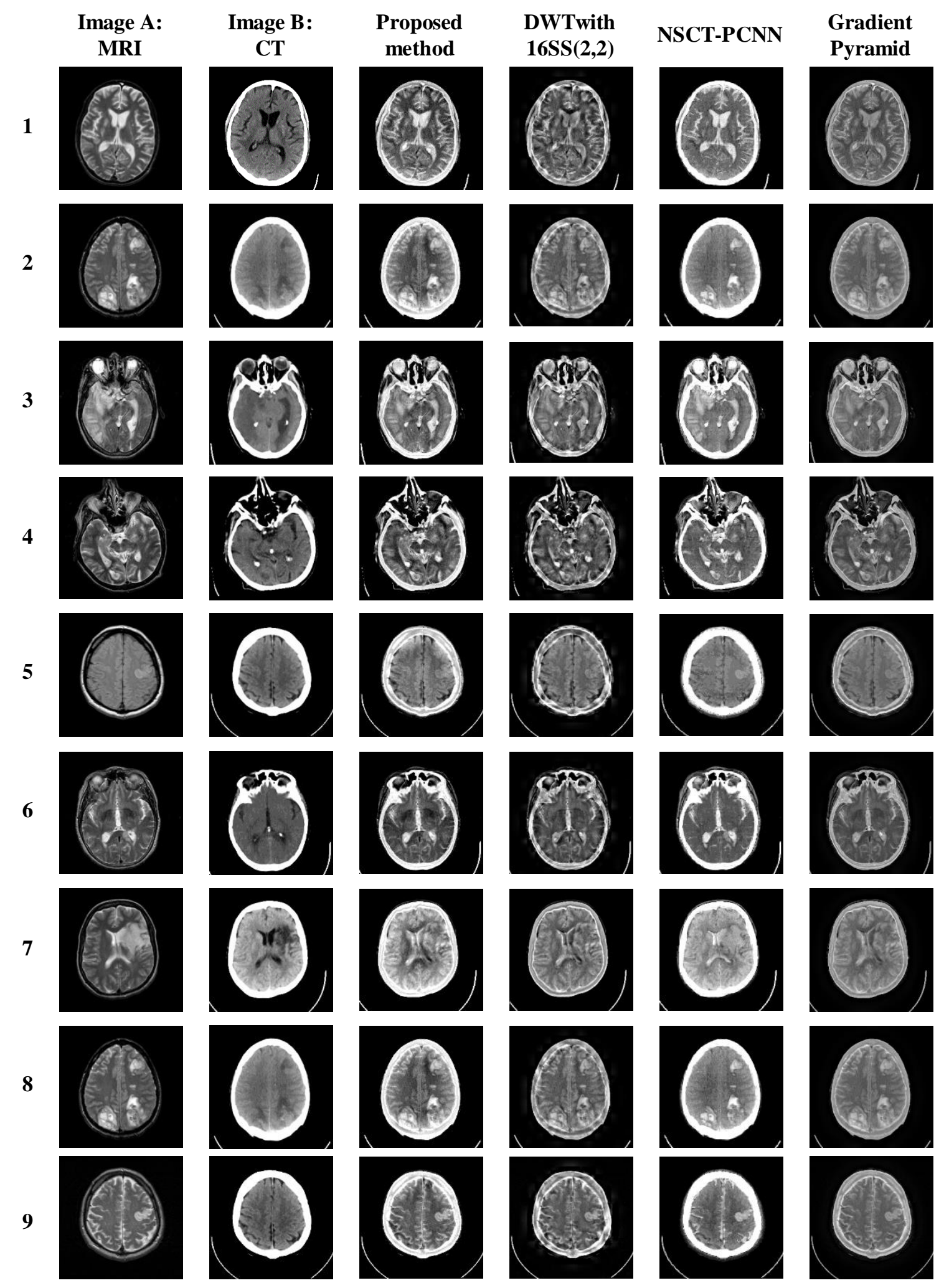

Fig.5. Performance comparisons using different methods.

The gradient pyramid has the distinguishing feature that each pyramid level generates only one band pass image. DWT is the most widely used for multi-resolution methods, it transforms images into limited direction. NSCT-PCNN method combined NSCT transform to decompose images and PCNN process to fused images. All those techniques decompose the input images into $L F$ and $H F$ sub-bands. In our evaluation, HF coefficients are combined using the maximum selection rule for all techniques where $L F$ coefficients are 
fused based on the PCNN model for the NSCT-PCNN method, the MPCNN for the proposed method and the average selection rule for DWT and Gradient pyramid techniques. Form Table 1 and Fig. 6, all groups of the proposed method provide a larger value of FF which indicates a better quality of the fused image. DWT also provides similar results with the proposed algorithm while NSCT-PCNN and Gradient pyramid algorithms provide similar results. Table 2 and Fig. 7 show the entropy results where it can be see that the proposed method gives the highest values for all groups than other methods which conclude that there are more information in our fused images. From Table 3 and Fig.8, we can see that our standard deviation results between the input registered images and the fused image are competitive with the NSCT-PCNN method for all groups that shows a good contrast compared to others as assessed by visual perception.

Table 1. Objective Evaluation FF

\begin{tabular}{|l|l|l|l|c|}
\hline FF & $\begin{array}{c}\text { PROPOSED } \\
\text { METHOD }\end{array}$ & DWT & SNCT-PCNN & GP \\
\hline G1 & 2,5156 & 2,4842 & 1,9885 & 1,9481 \\
\hline G2 & 2,9568 & 2,7623 & 1,6275 & 1,5843 \\
\hline G3 & 2,7955 & 2,7728 & 1,3692 & 1,4198 \\
\hline G4 & 2,6198 & 2,5625 & 1,2239 & 1,4198 \\
\hline G5 & 2,7888 & 2,5598 & 1,4876 & 1,4986 \\
\hline G6 & 2,9772 & 2,8118 & 2,2829 & 2,2593 \\
\hline G7 & 2,9271 & 2,8794 & 1,6046 & 1,5660 \\
\hline G8 & 2,9584 & 2,7590 & 1,6404 & 1,3601 \\
\hline G9 & 2,9562 & 2,9294 & 1,7272 & 1,6915 \\
\hline
\end{tabular}

Table2. Objective Evaluation E

\begin{tabular}{|l|l|l|l|c|}
\hline E & $\begin{array}{c}\text { PROPOSED } \\
\text { METHOD }\end{array}$ & DWT & SNCT-PCNN & GP \\
\hline G1 & 5,7978 & 5,3348 & 5,7154 & 5,4445 \\
\hline G2 & 5,4619 & 5,087 & 5,1693 & 5,0288 \\
\hline G3 & 5,8348 & 5,2839 & 5,4961 & 5,7458 \\
\hline G4 & 6,3061 & 5,6403 & 4,5524 & 5,7458 \\
\hline G5 & 5,4243 & 5,0084 & 4,0248 & 5,2877 \\
\hline G6 & 5,8185 & 5,2871 & 4,6477 & 5,5879 \\
\hline G7 & 5,5938 & 4,1140 & 5,0328 & 5,3348 \\
\hline G8 & 5,4379 & 5,0884 & 5,1548 & 5,0252 \\
\hline G9 & 5,7474 & 5,4287 & 5,1791 & 5,6201 \\
\hline
\end{tabular}

Table3. Objective SD

\begin{tabular}{|l|l|l|l|c|}
\hline SD & $\begin{array}{c}\text { PROPOSED } \\
\text { METHOD }\end{array}$ & DWT & SNCT-PCNN & GP \\
\hline G1 & 56,6536 & 47,5391 & 54,4109 & 41,786 \\
\hline G2 & 59,7953 & 46,1838 & 59,4096 & 42,9385 \\
\hline G3 & 58,2160 & 49,5622 & 56,5577 & 43,4314 \\
\hline G4 & 61,968 & 53,2342 & 61,7121 & 43,4314 \\
\hline G5 & 67,2034 & 54,8675 & 63,6761 & 49,3458 \\
\hline G6 & 57,6076 & 49,2818 & 56,1393 & 42,1374 \\
\hline G7 & 64,3792 & 51,7291 & 64,6975 & 46,3527 \\
\hline G8 & 59,8717 & 46,2108 & 59,4501 & 42,9612 \\
\hline G9 & 66,1388 & 56,5083 & 65,5791 & 49,8069 \\
\hline
\end{tabular}

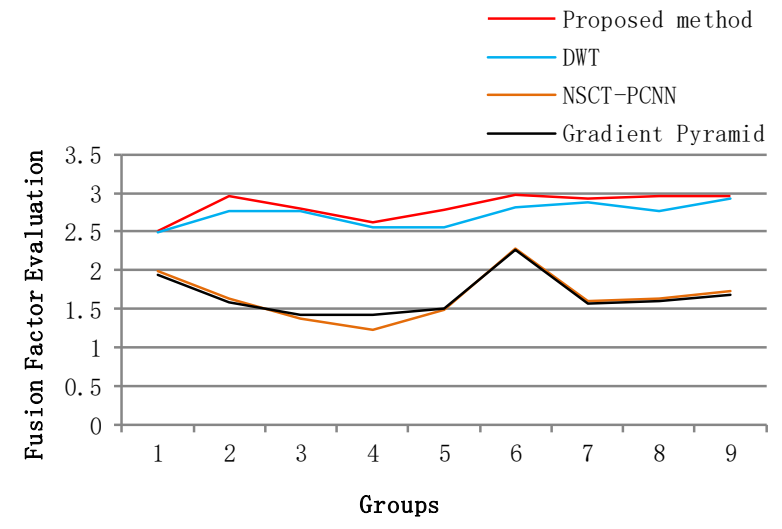

Fig. 6. Fusion factor evaluation.

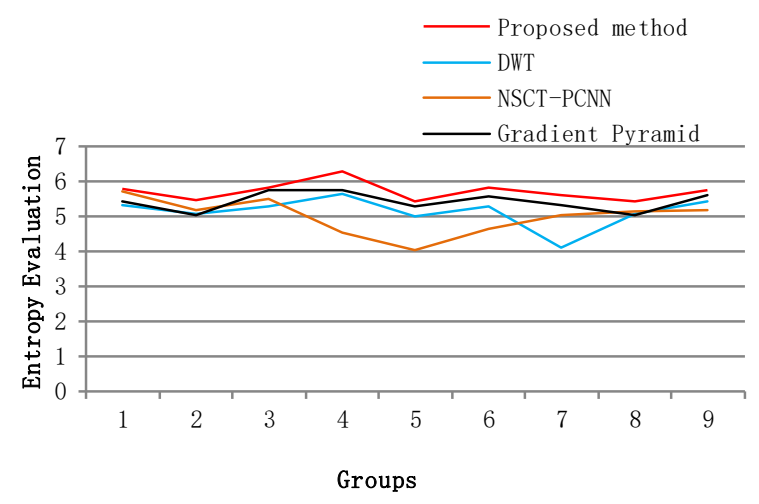

Fig. 7. Entropy evaluation.

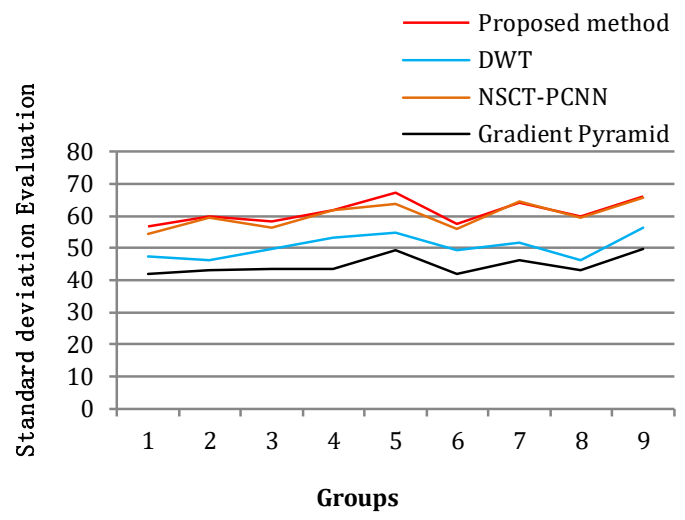

Fig. 8. Standard deviation evaluation. 
Table 4. Objective Evaluation $\mathrm{Q}^{\mathrm{AB} / \mathrm{F}}$

\begin{tabular}{|l|l|l|l|c|}
\hline Q & $\begin{array}{c}\text { PROPOSED } \\
\text { METHOD }\end{array}$ & DWT & SNCT-PCNN & GP \\
\hline G1 & 0,4118 & 0,4068 & 0,5313 & 0,4966 \\
\hline G2 & 0,4266 & 0,4687 & 0,5275 & 0,0208 \\
\hline G3 & 0,4230 & 0,4584 & 0,0397 & 0,0996 \\
\hline G4 & 0,4404 & 0,4600 & 0,0524 & 0,0996 \\
\hline G5 & 0,3310 & 0,4736 & 0,0248 & 0,0197 \\
\hline G6 & 0,4592 & 0,4949 & 0,6477 & 0,5687 \\
\hline G7 & 0,4339 & 0,5397 & 0,0353 & 0,0293 \\
\hline G8 & 0,4246 & 0,4693 & 0,0286 & 0,0187 \\
\hline G9 & 0,3572 & 0,4795 & 0,6251 & 0,5978 \\
\hline
\end{tabular}

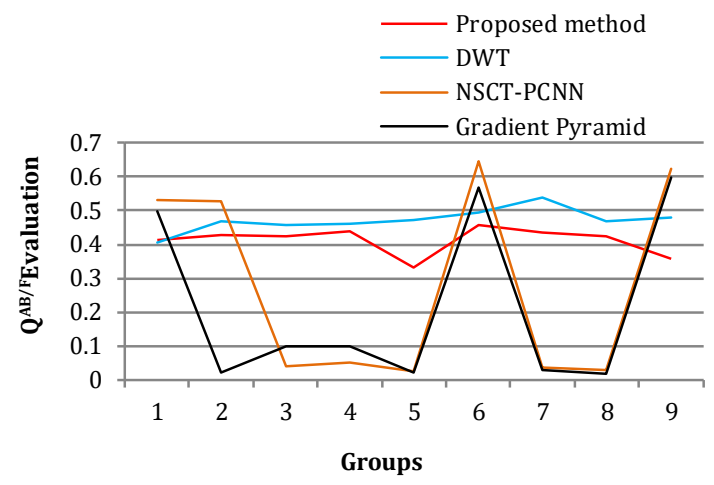

Fig. 9. Fusion quality index evaluation.

Most of the existing MIF methods suffer from several problems of image degradations such as contrast reduction, blocking effects and loss of image details. So, the edge information preservation presents one limit to the automatic process for MIF techniques due to the specific modality. Although, the best values of $\mathrm{Q}^{\mathrm{AB} / \mathrm{F}}$ are obtained by DWT for most image groups, the visual performance is not obviously as good as the proposed method as mentioned in Fig. 5. On the other hand, the proposed technique used the maximum selection method to fused HF coefficients that giving the image details. So, it will be suitable to improve HF fusion rules in the future works. As mentioned above, the entropy (E), the fusion factor (FF) and the Standard deviation (SD) values of the proposed method are the largest of all groups, which show that the performance is excellent in both subjective visual effect and objective evaluation criteria.

Table5. Computational Time Cost

\begin{tabular}{lcccc}
\hline \hline Methods & Proposed method & NSCT-PCNN & DWT & Pyramid gradient \\
\hline Time $(\mathrm{s})$ & 12 & 231 & 1 & 1 \\
\hline \hline
\end{tabular}

In addition, as we know that the time cost is an important criteria in clinical applications. So, we propose to compare only the critical time cost for each method. From Table 5, we conclude that the proposed method is faster than the NSCT-PCNN algorithm because NSCT transform computation is complex and time consuming, but slower than DWT and Pyramid gradient methods due to the learning procedure in the PCNN algorithm. Finally, we can draw the conclusion that the proposed method provides better performance result than other methods. It can be more flexible, robust and simpler than others fusion methods based on the PCNN model. But, it is applied only for grayscale medical images. So, more attention will be addressed to integrate the color components of the source images to enhance the performance of the proposed approach.

\section{Conclusion and Future Works}

In this paper, a novel fusion method is presented for MRI and CT registered images. The MSVD transform is proposed as a new tool for medical imaging fusion. The proposed method contains three parts. The MSVD transform is used to decompose images into $L F$ and $H F$ coefficients in the first part and to get the fused image in the last part. In the second part, the modified PCNN is applied to fuse $L F$ coefficients. It can adjust automatically the linking strength which is the important factor in our modified PCNN model. $H F$ coefficients are fused based on the maximum selection. Objective and subjective assessments reveal that the proposed method is simpler, more flexible and it could be well suited for medical real time applications. In future work, we will use new fusion rules for the proposed method. In addition, attempts will be made to enhance the performance of our algorithm to combine other medical images such as MRI and PET images which know an important evolution in the last years. 


\section{References}

[1] James, A., \& Dasarathy, B. V. (2014). Medical image fusion: A survey of the state of the art. Information Fusion, 19, 4-19.

[2] Kaur, A., \& Sharma, R. (2016). Review on image fusion algorithms. International Journal of Engineering Sciences, 17(1), 147-153.

[3] Shaveta, M., \& Arpinder, S. (2014). A comparative analysis of different image fusion techniques. International Journal of Computer Science, 2(1), 8-15.

[4] Kakarala, R., \& Ogunbona, P. O. (2001). Signal analysis using multiresolution form of the singular value decomposition. IEEE Transactions on Image processing, 10(5), 724-735.

[5] Gautam, S., \&Kumar, M. (2015). An effective image fusion technique based on multiresolution singular value decomposition. Infocomp Journal of Computer Science, 14(2), 31-43.

[6] Varsha, M., \& Kishore, M. R. (2014). Novel image fusion technique based on DWT \& MSVD.International Journal on Recent and Innovation Trends in Computing and Communication, 2(8), 2535-2540.

[7] Malini, S., \& Moni, R. S. (2015). Image denoising using multiresolution singular value decomposition transform. International Conference on Information and Communication Technologies. Procedia Computer Science, 46, 1708-1715.

[8] Naidu, V. P. S. (2011). Image fusion technique using multi-resolution singular value decomposition. Defence Science Journal, 61(5), 479-484.

[9] Subashini, M. M., \& Sahoo, S. K. (2014).Pulse coupled neural networks and its applications. International Journal of Expert Systems with Applications, 41, 3965-3974.

[10] Wang, Z., Wang, S., Zhu, Y., \&Ma, Y. (2015).Review of image fusion based on pulse-coupled neural network. Archives of Computational Methods in Engineering, 1-13.

[11] Yuan, Y., Jiang, M., \& Gao, W. (2014). Image fusion based on MPCNN and DWT in PCB failure detection. Computer Modelling and New Technologies, 18(7), 128-132.

[12] Biswas, B., Sen, B. K., \& Choudhuri, R. (2015). Remote sensing image fusion using PCNN model parameter estimation by gamma distribution in shearlet domain. Procedia Computer Science, 70, 304-310.

[13] Guo, Y., \& Huang, Y. (2016). A medical image fusion algorithm based on multi-channel PCNN in NSCT Domain. International Journal of Signal Processing, Image Processing and Pattern Recognition, 9(3), 367-382.

[14] Xu, X. Z., Wang, G. Y. Ding, S. F., Cheng, Y. H., \&Wang, X.S. (2016). Pulse coupled neural networks and parameter optimization methods. International Journal of Neural Computing and Applications, 1-11.

[15] Wang, Z., Wang, S., \& Zhu, Y. (2016). Multi-focus image fusion based on the improved PCNN and guided filter. Neural Processing Letters, 43, 1-20.

[16] Thakur, I., \&Saini, H. (2014). A Review: Analysis of SVD based image fusion methods. International Journal of Engineering Research and Technology, 3(5), 802-804.

[17] Kong, W.,Lei, Y.,\&Lu, X. N. (2011). Image fusion technique based on non-subsampled contourlet transform and adaptive unit-fast-linking pulse-coupled neural network. IET Image Processing, 5(2), 113-121.

[18] Chen, Y., Park, S. K., Ma, Y., \& Ala, R. (2011). A new automatic parameter setting method of a Simplified PCNN for image segmentation. IEEE Transactions on Neural Networks, 22(6).

[19] Biswa, B., Sen, B. K., \& Choudhuri, R. (2015). Remote sensing image fusion using PCNN model parameter estimation by gamma distribution in shearlet domain. Procedia Computer Science, 70, 304 $-310$.

[20] Zhan, K., Shi, J., Wang, H., Xie, Y., \& Li, Q. (2016). Computational mechanisms of pulse-coupled neural 
networks: A comprehensive review. Archives of Computational Methods in Engineering, 1-16.

[21] Chandana, M., Amutha, S., \& Naveen, K. (2014).A hybrid multi-focus medical image fusion based on wavelet transform. International Journal of Research and Reviews in Computer Science, 2(4).

[22] Petrovic, V. S., \& Xydeas, C.S. (2004). Gradient-based multiresolution image fusion. IEEE Transactions on Image Processing, 13(2), 228-237.

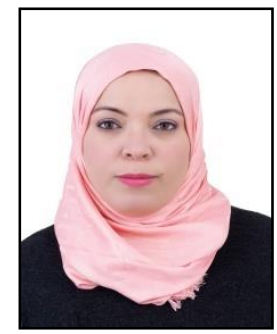

Hajer Ouerghi was born in Tunisia. She received an engineering degree in computer science and a master's degree in intelligent systems of imaging and artificial vision from the Higher Institute of Computer Science (ISI), Tunisia, in 2012 and 2013. She is currently working toward her Ph.D within LIMTIC Laboratory at ISI in Tunisia. Her research interests include image processing, image fusion and computer vision.

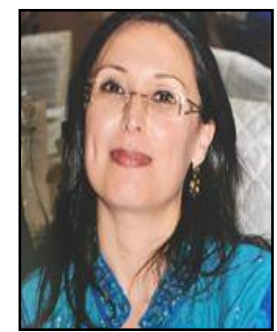

Olfa Mourali received his Ph.D from Higher Institute of Management of Tunis (ISG) in Tunisia. She is currently an assistant professor in the Department of Software Engineering and Information Systems at Higher Institute of Computer Science (ISI). She is member of LIMTIC Research Laboratory at ISI. She worked as a director of studies and internships at ISI, Tunisia, in 2013. Her research interests cover image treatment and fusion, big data, and management of uncertainty with Bayesian networks.

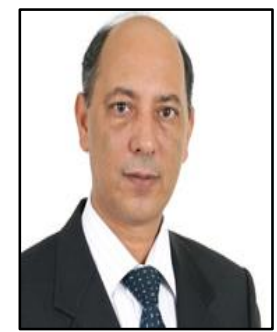

Ezzeddine Zagrouba received his HDR from FST/University Tunis ElManar and his Ph.D and engineering degree from the Polytechnic National Institute of Toulouse (ENSEEIHT/INPT) in France. He is a professor at the Higher Institute of Computer Science. He is a vice president of the Virtual University of Tunis and the director of LimTic Laboratory. His main activity is focused on intelligent imaging and computer vision. 